\title{
Post-print: Near Axisymmetric Partial Wetting Using Interface-Localized Liquid Dielectrophoresis
}

\author{
Zuzana Brabcova,* Glen McHale and Gary G.Wells \\ Smart Materials and Surfaces Laboratory, Faculty of Engineering \& Environment, Northumbria University, \\ Ellison Place, Newcastle upon Tyne, NE1 8ST, United Kingdom \\ Carl V. Brown, Michael I. Newton, Andrew M.J. Edwards \\ School of Science and Technology, Nottingham Trent University, Clifton Lane, Nottingham, NG11 8NS,
}

\section{Published as Langmuir 32(42), pp 10844-10850 (2016) \\ http://dx.doi.org/10.1021/acs.langmuir.6b03010}

\begin{abstract}
The wetting of solid surfaces can be modified by altering the surface free energy balance between the solid, liquid, and vapour phases. Liquid dielectrophoresis (L-DEP) can produce wetting on normally non-wetting surfaces, without modification of the surface topography or chemistry. L-DEP is a bulk force acting on the dipoles of a dielectric liquid and is not normally considered to be a localized effect acting at the interface between the liquid and a solid or other fluid. However, if this force is induced by a non-uniform electric field across a solid-liquid interface, it can be used to enhance and control the wetting of a dielectric liquid. Recently, it was reported theoretically and experimentally that this approach can cause a droplet of oil to spread along parallel interdigitated electrodes thus forming a stripe of liquid. Here we show that by using spiral shaped electrodes actuated with four $90^{\circ}$ successive phase shifted signals, a near axisymmetric spreading of droplets can be achieved. Experimental observations show that the induced wetting can achieve film formation, an effect not possible with electrowetting. We show that the spreading is reversible thus enabling a wide range of partial wetting droplet states to be achieved in a controllable manner. Furthermore, we find that the cosine of the contact angle has a quadratic dependence on applied voltage during spreading and deduce a scaling law for the dependence of the strength of the effect on the electrode size.
\end{abstract}




\section{INTRODUCTION}

The wetting of solid surfaces is of interest to a wide range of disciplines and has many applications, such as creating thin films, surfaces coating, adhesion, droplet deposition and droplet control. There are many ways to modify the wetting properties of solid surfaces, all based on altering the energy balance between the solid, liquid and vapour phase. ${ }^{1}$ It can be achieved either by changing the properties of the liquid ${ }^{2,3}$ or the solid surface, ${ }^{4}$ or by introducing an electric field. ${ }^{5}$ In recent years, uniform electric fields have proved important in manipulating $^{6}$ and controlling droplets ${ }^{7}$ of conducting liquids. Using the liquid-solid contact area as one electrode, electrowetting ${ }^{8-11}$ reversibly increases the hydrophilicity of the solid surface and reduces the contact angle of a droplet of a conductive liquid without altering the surface chemistry or the liquid properties. Thus, a droplet can be shaped or moved leading to applications ${ }^{12}$ such as liquid lenses, ${ }^{13-15}$ liquid optical elements, ${ }^{16,17}$ microfluidics ${ }^{6}$ or droplet manipulation. ${ }^{7,18}$ However, there are significant limitations to the electrowetting approach including the need for any liquids of interest to be conducting and normally the need for direct electrical contact with the liquid, although there are examples of using non-contacting co-planar electrodes. ${ }^{19,20}$ Also an inability to spread a droplet to a film state, due to the existence of a minimum saturation contact angle is a disadvantage. ${ }^{21}$ Typically the minimum contact angle is $\sim 60^{\circ}$ for a DC voltage or $45^{\circ}$ for an AC voltage, and is due to the unexplained effect of contact angle saturation. ${ }^{22,23}$ All of these factors limit the range of applicability of the electrowetting approach. ${ }^{24}$

Liquid dielectrophoresis (L-DEP) is the attraction of polarized dipoles into regions of relatively stronger electric fields and is a driving force for motion of bulk insulating dielectric liquids. This is because a non-uniform electric field generates unequal forces on dipoles that result in a dielectrophoretic (DEP) force causing movement of the liquid into areas of the highest gradient of the electric field..$^{8,25-29}$ For conducting liquids, Jones has discussed the 
relationship between electrowetting and the electromechanical response of the liquid, for which the high frequency limit, equivalent to an insulative liquid, is liquid dielectrophoresis. ${ }^{9}$ For example, deionized water $\left(\sim 10^{-4} \mathrm{~S} / \mathrm{m}\right)$ in air behaves as a conductive liquid for frequencies below $60 \mathrm{kHz}$ and as a dielectric liquid for frequencies above $60 \mathrm{kHz} .^{9}$

Recently, we have shown how an interface localized form of liquid dielectrophoresis ${ }^{8}$, can be used to create spreading and superspreading of droplets of a dielectric liquid in air ${ }^{26,30,31}$ or a second liquid. ${ }^{32}$ This approach, described as dielectrowetting, enables the control of the oleophobicity of materials by applying a voltage. Dielectrowetting utilizes the dielectric properties of liquids, but the effect is localized to the interface ${ }^{31}$ and differs significantly from electrowetting. ${ }^{11}$ With a suitable decaying electric field, the effect of L-DEP can be bounded to the solid-liquid or liquid-vapor interface and can be used to induce spreading or superspreading of droplets of non-conducting liquids. ${ }^{30,31}$ Moreover, the saturation of contact angle appears lower than in the case of electrowetting ${ }^{30}$ and a significant difference is in achieving the full range of wetting from the droplet to the film state that has not been reported in the case of electrowetting. ${ }^{22-24}$ Full wetting has been shown to be useful for optical applications based on controlled changes to the shapes of films of liquids. ${ }^{16,17,31}$

In wetting the Young's law contact angle for a partially wetting droplet can be obtained by considering the surface free energy changes as a contact line advances over a small area, $\Delta A$. Such an advance removes solid-vapor interfacial area and replaces it with solid-liquid interfacial area thus causing a change in the surface free energy of $\left(\gamma_{S L}-\gamma_{S V}\right) \Delta A$ where $\gamma_{S L}$ and $\gamma_{S V}$ are the solid-liquid and solid-vapor interfacial tensions, respectively. An additional surface free energy of $\cos \theta\left(\gamma_{L V} \Delta A\right)$ is also created. In equilibrium, the total change in surface free energy, $\Delta F=\left(\gamma_{S L}-\gamma_{S V}\right) \Delta A+\cos \theta\left(\gamma_{L V} \Delta A\right)$ vanishes and so the equilibrium contact angle is $\cos \theta_{e}=\left(\gamma_{S V}-\gamma_{S L}\right) / \gamma_{L V}$, which is Young's law. In the classic electrowetting (on dielectric) 
arrangement, the droplet is composed of a conducting liquid and rests on the top of smooth solid dielectric of thickness $d_{\text {ins }}$ and permittivity $\varepsilon_{o} \varepsilon_{r}$, which has a uniform electrical contact on its bottom surface. ${ }^{9-11}$ Thus, the spatial extent of the solid-liquid contact area defines an electrode and the structure provides a capacitive energy contribution, $\varepsilon_{o} \varepsilon_{r} V^{2} / 2 d_{\text {ins }}$ per unit area, where $V$ is the applied voltage, to the energy balance. The cosine of the equilibrium contact angle is then modified to include a term representing the balance of capacitive energy to liquid-vapor interfacial energy,

$$
\cos \theta(V)=\cos \theta(0)+\left(\frac{\varepsilon_{o} \varepsilon_{r}}{2 \gamma_{L V} d_{i n s}}\right) V^{2}
$$

In liquid-dielectrophoresis energy is stored in the liquid due to the polarization of dipoles by the non-uniform electric field. A surface fabricated interdigitated electrode structure with electrodes and gaps of size $d$ (in our work electrode width is always equal to the gap size) and an applied sinusoidal voltage generates an electric field decaying away from the surface (assumed here to be the z-axis) in an exponential manner. The penetration depth, $\delta$, is defined via $V(\mathrm{z})=V_{0} \mathrm{e}^{-2 z / \delta}$ and is proportional to $d$ according to the stripe theory of reference $\mathrm{e}^{26}$. In a similar manner to electrowetting this results in a modification of the cosine of the equilibrium contact angle to include a term representing the balance of dielectrophoretic energy to liquidvapor interfacial energy, ${ }^{33}$

$$
\cos \theta(V)=\cos \theta(0)+\left(\frac{\varepsilon_{o}\left(\varepsilon_{l}-1\right)}{2 \gamma_{L V} \delta}\right) V^{2}
$$

where $\varepsilon_{o} \varepsilon_{l}$ and $\delta$ are the permittivity of the dielectric liquid and penetration depth of the electric field into the liquid. Thus, the periodicity of the surface fabricated electrodes determines a surface layer of the liquid of effective thickness, $\delta$ (Figure 1a), which is analogous to the thickness of the solid dielectric in electrowetting and justifies the term 
interface-localized liquid dielectrophoresis. When the localization of the effects of liquid dielectrophoresis is used with droplets of height larger than $\delta$ and controls droplet spreading, rather than the liquid-vapor interface on the surface of a liquid film, the effect has been called dielectrowetting. ${ }^{30}$

Thus far interface localized L-DEP has been primarily used for spreading droplets of nonconducting liquids along parallel interdigitated electrodes (IDEs) into a non-axisymmetric stripe-shaped droplet form. Most recently, some of the present authors have shown that it is possible to use L-DEP to initiate an axisymmetric (circular) film and observe the dynamics of its dewetting back into a macroscopic equilibrium droplet that existed prior to the application of any voltage. ${ }^{34}$ An outstanding challenge is to create spreading of an axisymmetric droplet with the ability to programme, using a voltage, any desired axisymmetric partially wetting droplet state concluding with a film state. Achieving axisymmetric droplet shapes is essential to convert the idea of L-DEP induced partial wetting from an interesting insight into greater practical relevance, for example in terms of liquid lenses. ${ }^{14}$ The difficulties in doing so arise from the fact that an AC voltage on parallel IDEs ${ }^{30}$ create a non-uniform periodic field with maximum values located at the electrode edges. ${ }^{35}$ The resulting periodic energy barriers due to the electrodes prevent the droplet spreading axisymmetrically and favour spreading along the electrodes rather than across them. Overcoming these energy barriers is the main challenge to achieving axisymmetric droplet spreading. In this work, we present a method for achieving near axisymmetric spreading of droplets using a phase-shift signal activation that differs from previous works, ${ }^{26,30-32,36}$ and a spiral pattern electrode design that specifically shapes the droplet. We also show experimentally that the change in the cosine of contact angle has a quadratic dependence on voltage in a similar manner to electrowetting and the stripe form of L-DEP induced wetting. Moreover, the strength of the effect of the 
dielectrowetting is found to obey a scaling law related to the size of the electrodes although the exact form differs from that found for the dielectrowetting using parallel IDEs.

\section{EXPERIMENTAL SECTION}

Achieving axisysmetric spreading using an anistropic liquid such as a liquid crystals has been attempted in the literature. ${ }^{37}$ However, for dielectric liquids with isotropic properties initial attempts at axisymmetric spreading using spiral and circular arc electrode designs have shown non-ideal faceting of the droplets edge. ${ }^{38}$ We therefore looked for inspiration to biological separation methods where spiral electrodes are used, but with a four-phase voltage actuation for travelling wave particle dielectrophoresis that creates a rotating electric field. ${ }^{35,39,40}$ Our hypothesis was that a rotating electric field electrode design would be less likely to have energy barriers inducing faceting. To investigate axisymmetric L-DEP induced spreading of droplets, a prototype device was designed and fabricated based on electrode designs used for biological separations. ${ }^{40}$

Each electrode pattern contained four spiral electrodes of width $d$, separated equidistantly by gaps of the same dimension, $d$, as the electrode width (Figure 1b) produced using a standard photolithographic etch-back technique. In biological separation experiments these four spiral electrode elements are actuated with sinusoidal signals with successive relative phase differences of $90^{\circ}$ to generate a rotating electric field. Because the penetration depth of the electric field into the liquid is expected to be directly related to the electrode geometry, samples with electrode sizes of $d=40,60,80,100,120$ and $180 \mu \mathrm{m}$ were studied. The thickness of the gold electrodes was $100 \mathrm{~nm}$ and the surfaces, including electrodes, were covered with a $1.7 \mu \mathrm{m}$ capping layer of photoresist SU8-2002 (MicroChem) which can withstand $\sim 550 \mathrm{~V}$ before electrical breakdown. This treatment provides a thin dielectric layer with a uniform smooth surface to the liquid and also ensures electrolysis and subsequent destruction of a device does not occur if the droplet becomes conductive for any reason (e.g. 
when using hygroscopic liquids). To achieve a high initial contact angle ( $\left.>90^{\circ}\right)$, the substrate was coated with a commercial solution Novec 1700 (3M) providing an oleophobic surface.

Figure 1c shows the experimental setup. Initially, $1 \mathrm{kHz}$ sinusoidal signals with phase shifts of $0^{\circ}$ and $90^{\circ}$ were provided by a waveform generator (SoundBlaster Omni Surround 5.1) connected to two amplifiers (TREK, PZD700A). The ground was decoupled via transformers and amplified signals were fed into a phase shifter where each signal was split so that one part of the original signal was kept unchanged, and the other one was phase shifted by $180^{\circ}$ (inverted). Using this method it was possible to create a four-phase approach with four signals with relative phase shifts of $0^{\circ}, 90^{\circ}, 180^{\circ}$ and $270^{\circ}$. Each signal was then connected to one of the four spirals within the electrode pattern. Droplets of glycerol were deposited onto the electrodes (typical volume of $5 \mu \mathrm{l}$ ) and both side and top view images were recorded. Since pure glycerol is a hygroscopic liquid (99.5\%, Sigma Aldrich) it was stored in a perfectly sealed container and a fresh droplet was used for every experiment. We also verified that similar results occurred at a higher frequency of $2 \mathrm{kHz}$ to ensure the liquid was exhibiting a dielectric response consistent with expectations of operating above a cross-over frequency of $14 \mathrm{~Hz}$ for dielectrophoresis to dominate that arises from a permittivity of 42.5 and conductivity of $5.6 \times 10^{-8} \mathrm{~S} / \mathrm{m}^{9} \quad$ The top view images were captured with a standard Canon camera D600 fitted with a macro objective (Carl-Zeiss Jena, Pancolar). Side profile view images were captured using a Krüss Drop Shape Analysis system (DSA30) that also enabled the measurement of the contact angles by fitting an ellipse to the droplet profile. Thus, all contact angles discussed in this work are macroscopic ones rather than microscopic ones, which may differ at length scales significantly smaller than the electric field penetration depth set by the electrode size. The advancing contact angle of the glycerol droplet on the surface measured by the volume addition method was $104^{\circ}$. 

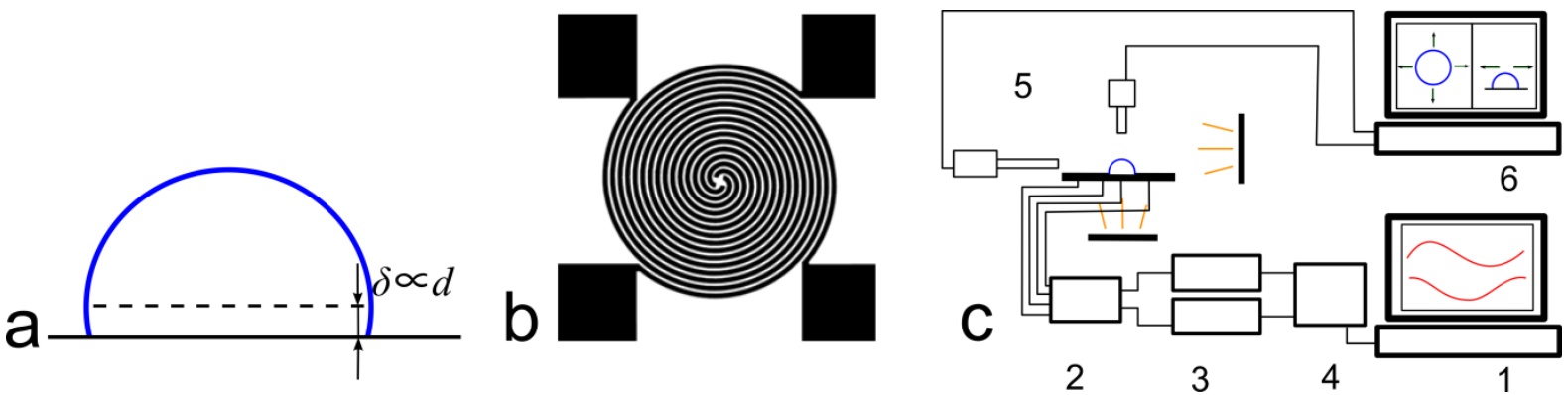

Figure 1. (a) Schematic of a droplet with the penetration depth, $\delta$. of the electric field into the liquid illustrated and indicating its proportionality to electrode size, $d$, (b) Four arm spiral electrode design with electrode width and spacing between electrodes both of size $d$; (c) Schematic of the experimental setup: 1 software signal generating, 2 waveform generator (soundcard), 3 amplifiers, 4 ground decoupler and phase shifter, 5 imaging and light system, 6 image capture.

\section{RESULTS AND DISCUSSION}

We performed initial testing using voltages phase shifted by $0^{\circ}$ and $180^{\circ}$ applied to every other electrode to understand how spreading of glycerol might differ from actuation using the four-phase approach with successively $90^{\circ}$ phase-shifted voltages. We also tested spreading used an alternative approach with ground and voltage actuation rather than $0^{\circ}$ and $180^{\circ}$. In these initial tests with these electrode designs using actuation voltages with $0^{\circ}$ and $180^{\circ}$, with and without the $0^{\circ}$ held at ground, film spreading was not achieved. Typically, a minimum contact angle of around $50^{\circ}$ was observed and higher applied voltages did not result in further spreading (Figure S1).

Figure 2 shows top view images of droplets sitting on an electrode pattern of electrode size $d=60 \mu \mathrm{m}$ with the voltage of $300 \mathrm{~V}$ (rms). From Figure 2a to Figure 2c, the pattern actuation was follows (anti-clockwise starting with the left bottom electrode pad): voltage-groundvoltage-ground, phase shifted signals of $0^{\circ}-180^{\circ}-0^{\circ}-180^{\circ}$, and phase shifted signals of $0^{\circ}-90^{\circ}-$ $180^{\circ}-270^{\circ}$, respectively. A small distortion from a circular axisymmetric shape was observed in all cases consistent with a spreading along the spiral structure, with the closest approximation to axisymmetry occurring for the four-phase approach (Figure 2c). Moreover, only the 4 phase shifted signal approach provided full spreading of the droplet into the film. 


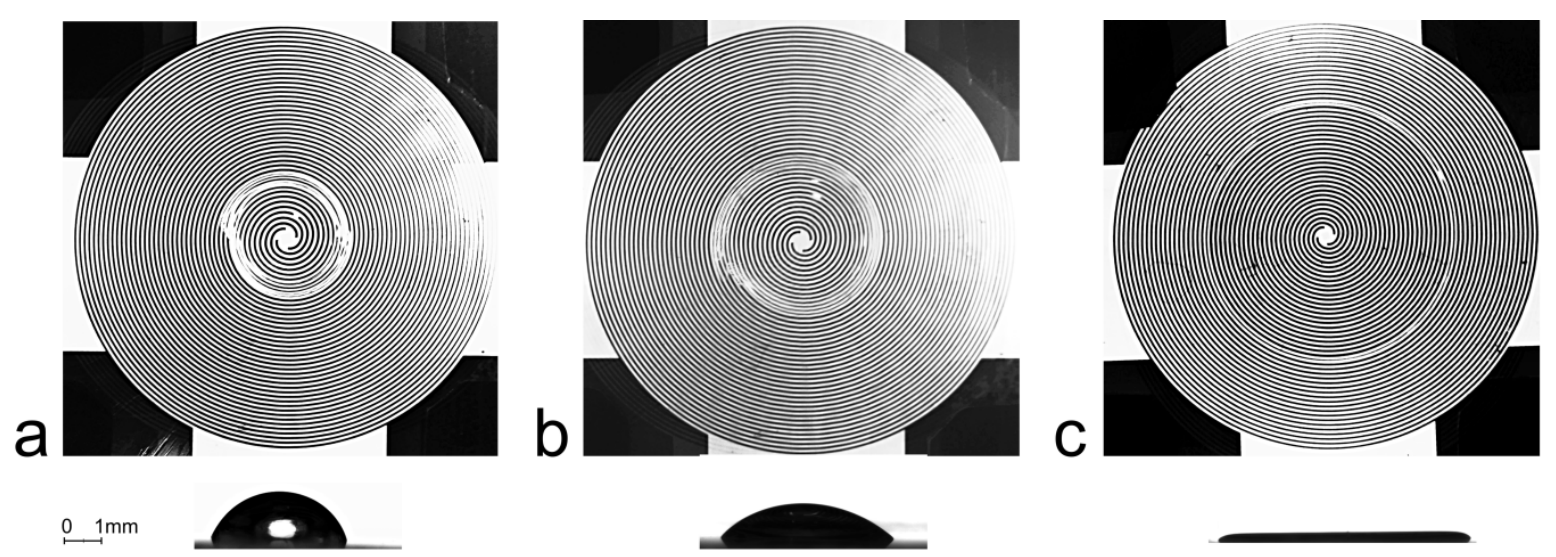

Figure 2. Different actuation of the spiral electrode array $d=60 \mu \mathrm{m}, V_{\mathrm{rms}}=280 \mathrm{~V}$ (a) voltage-ground. (b) signals of phase shifts of $0^{\circ}$ and $180^{\circ}$. (c) signals of phase shifts of $0^{\circ}, 90^{\circ}, 180^{\circ}, 270^{\circ}$.

We now focus on experiments using only the four-phase actuation method. In these experiments, the initial side profile shape of our deposited droplets before application of a voltage is slightly deformed from a circular arc. This is consistent with expectation given the capillary length for glycerol $\kappa^{-1}=\left(\gamma_{L V} / \rho g\right)^{1 / 2}=2.3 \mathrm{~mm}$, where $\gamma_{L V}$ is the surface tension, $\rho$ the density and $g$ the acceleration due to gravity, and our droplets have an initial (zero applied voltage) contact radius of $r_{o} \sim 1.15 \mathrm{~mm}$ resulting in a ratio $r_{o} / \kappa^{-1} \sim 0.5$. Nonetheless, the contact angle at the contact line is independent of shape and, taking the droplet shown in Figure $\mathbf{3}$ on a $d=100 \mu \mathrm{m}$ electrode pattern as a typical example, it was found to be $\theta(V=0)=102 \cdot 0^{\circ} \pm 0.7^{\circ}$. Viewed from above, at zero voltage the droplet shows a circular outline indicating a good initial axisymmetry. When the voltage is increased, the liquid is driven along the spiral electrodes resulting in an increased contact radius. The initial movement looks like a slight adjustment of the side view profile of the droplet with the contact angle falling from $102^{\circ}$ to $90^{\circ}$. From the top view the contact line motion along the spiral is visible and this gives a distortion of the axisymmetry related to the spatial resolution of the electrode pattern defined by the size of the electrode width and gap. With increasing voltage, the droplet continues a spreading motion along the spiral towards the edges of the electrode pattern. This spreading 
increases the contact diameter whilst reducing the vertical height of the droplet. When observed from the side profile the contact angle of the droplet decreases. Eventually the voltage causes the droplet to fully spread into a film that appears to retain an almost circular shape when viewed from above. This reduction in observed contact angle continues to angles well below the saturated contact angle usually observed in electrowetting. However, as the contact angle reduces to less than $\sim 15^{\circ}-20^{\circ}$ the thin spreading film of liquid appears to retain a finite contact angle localized at the edge of the film. ${ }^{31,32}$ This general spreading behaviour with applied voltage was observed in all experiments on all electrode patterns irrespective of the precise value of the spiral width and gap $d$.
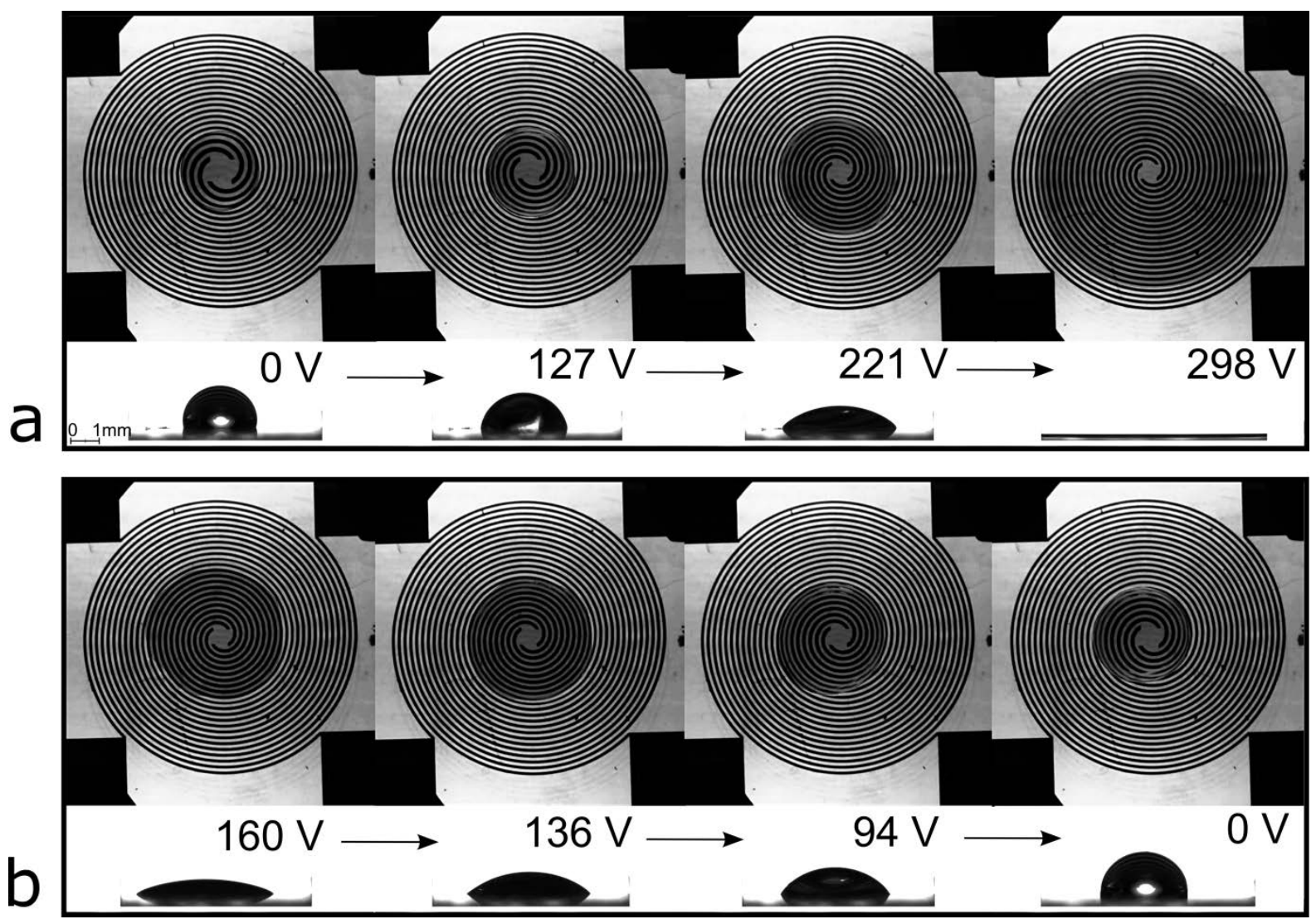

Figure 3. Top and side view images of a droplet of glycerol on spiral electrode pattern of width and gap spacing of $d=100 \mu \mathrm{m}$ showing the effect of (a) increasing and (b) decreasing voltage (rms). 
Figure 4 shows both an increasing voltage half cycle and a decreasing voltage half-cycle for a droplet on a $d=100 \mu \mathrm{m}$ electrode pattern plotted using the root mean square voltage, $V_{\text {rms; }}$; the data showing dielectrowetting can achieve contact angles well below the electrowetting saturation minimum is evident in this data. The top-view droplet shape changes are smooth for the increasing voltage half cycle, but involve jumps during the decreasing voltage halfcycle as the droplet retracts (Figure $4 \mathrm{~b}, \mathrm{c}$ ). The solid curve is a fit to a quadratic voltage dependence of $\cos \theta$ during the increasing voltage half-cycle for contact angles above $20^{\circ}$; this is shown by the inset in Figure 4a. The initial data point before the voltage was applied lies above the trend of the decreasing contact angle with the increasing voltage. This is likely to be due to the deposition method of the droplet and the initial contact angle being defined by contact angle hysteresis. ${ }^{41}$ A similar effect is visible when the voltage is changed from increasing to decreasing and the contact angle changed from a receding contact angle to an advancing contact angle. Eventually as the voltage is remove entirely the droplet regains a circular shape when viewed from above and achieves a contact angle of $\sim 80^{\circ}$. An example of droplet spreading for repeated voltage cycles are shown in the supplementary information (Figure S3). In the remainder of this section, we focus on the changes in contact angle as the voltage increases during the first applied half-cycle, which were always observed to be smooth changes in droplet shape. 

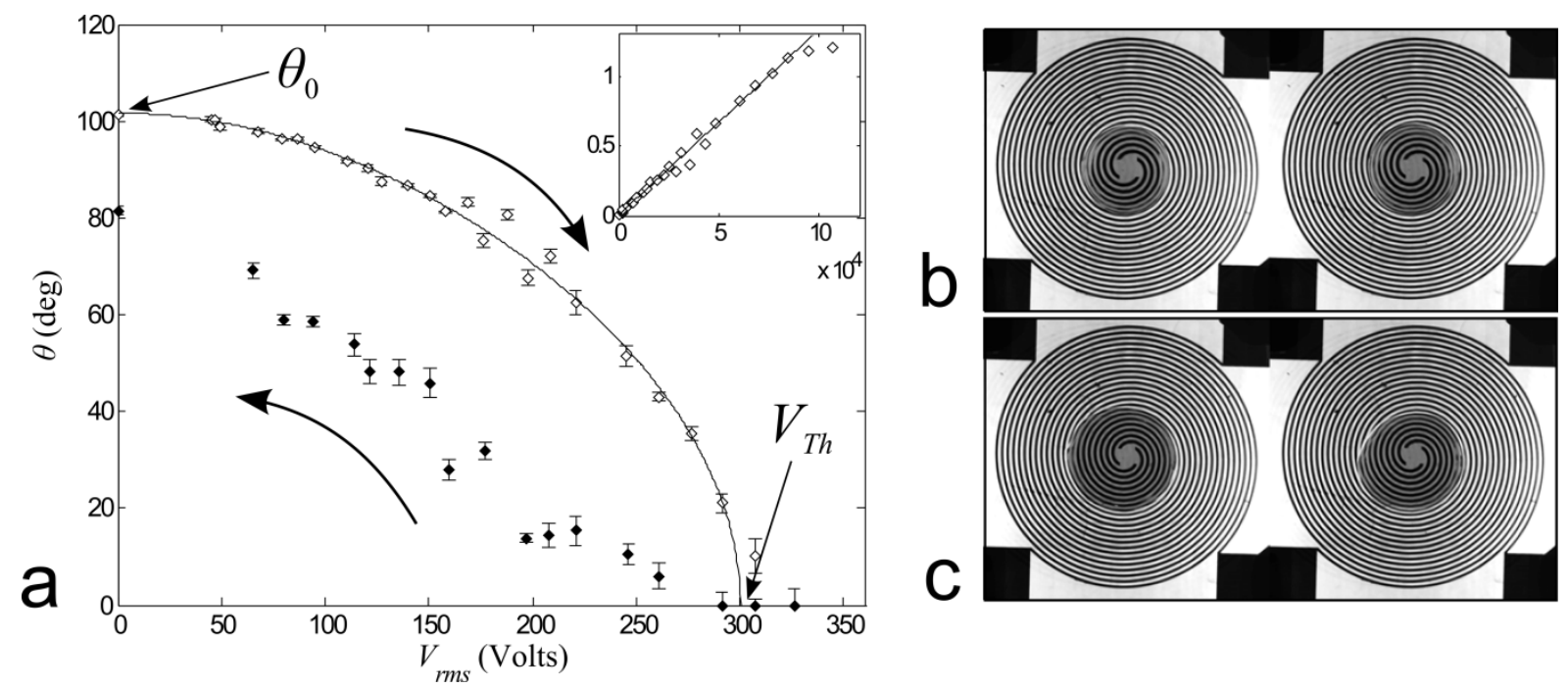

Figure 4. (a) The contact angle-voltage relationship; open and filled circles represent the increasing and decreasing voltage half-cycles, respectively. The height of the droplet goes from $1.52 \mathrm{~mm}$ to 0.08 $\mathrm{mm}$ with the increasing voltage half cycle (Figure S2). Inset: data plotted as $\Delta \cos \theta=\cos \theta\left(V_{r m s}\right)$ $\cos \theta(0)$ versus (rms) voltage squared; the solid line is the fit using data with contact angles above $20^{\circ}$. (b) Two consecutive top view images of a droplet during the increasing voltage half-cycle showing the smooth change in a shape. (c) Two consecutive top view images of a droplet during decreasing voltage half-cycle showing discontinuous jumps as the droplet retracts.

The experimentally observed quadratic dependence on voltage, $V_{r m s}$, of $\Delta \cos \theta\left(V_{r m s}\right)=\cos \theta\left(V_{r m s}\right)-\cos \theta(0)$ in the inset to Figure 4 is consistent with theoretical expectations from both electrowetting and for dielectrowetting of stripe droplets. ${ }^{30,31,36}$ Such a straight line relationship can be characterized by two parameters, which are usually the slope and intercept. The slope represents the strength of the effect from the electric field and for electrowetting, according to eq. (1), is determined by the ratio of the permittivity to thickness of solid dielectric multiplied by the liquid-vapor interfacial tension, $\varepsilon_{o} \varepsilon_{r} / 2 \gamma_{L V} d_{i n s}$. In the dielectrowetting case, according to eq. (2), the strength is determined by the ratio of the permittivity of the liquid minus that of air to the penetration depth of the electric field multiplied by the solid-vapor interfacial tension, $\varepsilon_{o}\left(\varepsilon_{l}-1\right) / 2 \gamma_{L V} \delta$. Thus, the slope represents the change of stored capacitive energy (electrowetting) or dielectrophoretic energy (dielectrowetting) to the solid-liquid interfacial energy. 
For the case of straight planar parallel interdigitated electrodes the penetration depth scales with the periodicity of the electrodes, which for equal width electrodes to gaps both of size $d$, and so gives $\varepsilon_{o}\left(\varepsilon_{l}-1\right) / 2 \gamma_{L V} \delta \propto \varepsilon_{o}\left(\varepsilon_{l}-1\right) / 2 \gamma_{L V} d{ }^{30}$ Thus in both cases, the strength of the voltageinduced effect has an inverse dependence on a size either of the insulator thickness, $d_{i n s}$, or the electrode gap, $d$. In both cases, the intercept for the straight line plot for $\cos \theta\left(V_{r m s}\right)$ is given by the cosine of the initial contact angle, $\cos \theta(0)$, prior to application of a voltage. However, when analysing plots of the type of Figure 4, it is convenient to use a parameter, $V_{T h}$, representing the threshold voltage at which the contact angle would vanish, i.e. $\cos \theta\left(V_{T h}\right)=1$, rather than the slope, together with the cosine of the initial contact angle, $\cos \theta(0) \cdot{ }^{31}$ In this notation the slope is defined as $\left(1-\cos \theta_{0}\right) / V_{T h}{ }^{2}$ where $\theta_{0}$ is the defined by $\cos \theta_{0}=\cos \theta\left(V_{r m s}=0\right)$. The similarity between equations (1) and (2) can then be seen as experimental results for both electrowetting and dielectrowetting can be analyzed using an equation with similar format ${ }^{31}$, the derivation of equation (3) is provided in the Supplementary Information.

$$
\cos \theta\left(V_{r m s}\right)=\cos \theta_{0}+\left(1-\cos \theta_{0}\right)\left(\frac{V_{r m s}}{V_{T h}}\right)^{2}
$$

This allows an experimental test of the quadratic dependence on (rms) voltage, $V_{r m s}$, of $\cos \theta\left(V_{r m s}\right)$ and a determination of the strength of the voltage-induced effect, (1- $\left.\cos \theta_{0}\right) / V_{T h}{ }^{2}$ via its dependence on two controlling parameters $\theta_{0}$ and $V_{T h}$.

To test the validity of equation (3) for the four-phase activation of our spiral electrode pattern designs, Figure 5 plots the full set results for all spiral electrode pattern sizes $d=40-180 \mu \mathrm{m}$. In each case, the inset plots $\Delta \cos \theta=\cos \theta\left(V_{r m s}\right)-\cos \theta_{0}$ as function of the square of the rms voltage, $V_{r m s}{ }^{2}$, and therefore tests its linearity. In each case the linear fit has been taken over 
all data points above $\theta=20^{\circ}$ and these fits are then displayed as the solid curves in the main figure in each panel.
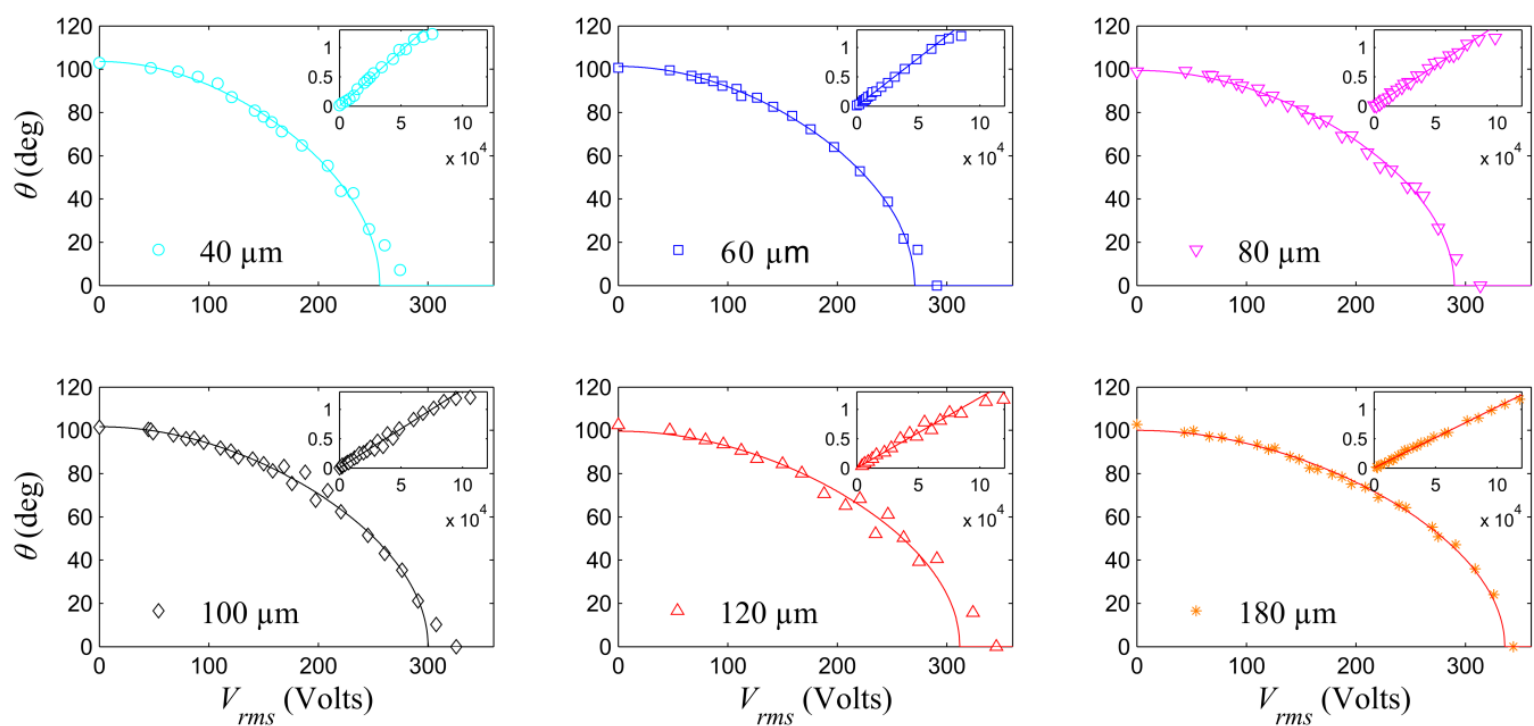

Figure 5. The contact angle-voltage relationship for the increasing voltage half-cycles for all electrode sizes $(d=40,60,80,100,120,180 \mu \mathrm{m})$. Inset: data plotted as $\Delta \cos \theta=\cos \theta\left(V_{r m s}\right)-\cos \theta(0)$ versus (rms) voltage squared; the solid lines are fits using data with contact angles above $20^{\circ}$.

The controlling experimental parameters for these experiments on the spiral electrode pattern design and the parameters deduced from the experiments are summarized in Table 1.

Table 1. Controlling experimental parameters $\left(\theta_{0}\right.$ and $\left.V_{\mathrm{Th}}\right)$ and parameters $\left(\cos \theta_{0}\right.$ and 1$\left.\cos \theta_{0}\right) / V_{T h}^{2}$ deduced from data in Fig. 5.

$\begin{array}{lcccccc}\boldsymbol{d}(\boldsymbol{\mu m}) & \mathbf{4 0} & \mathbf{6 0} & \mathbf{8 0} & \mathbf{1 0 0} & \mathbf{1 2 0} & \mathbf{1 8 0} \\ \theta_{0}(\mathrm{deg}) & 103.6 \pm 2.2 & 101.3 \pm 1.2 & 99.4 \pm 1.7 & 101.7 \pm 1.7 & 99.6 \pm 1.5 & 100.0 \pm 1.1 \\ V_{T h}(\text { Volts }) & 256.0 \pm 1.9 & 270.7 \pm 1.8 & 289.8 \pm 0.9 & 300.1 \pm 1.0 & 312.0 \pm 1.2 & 336.0 \pm 1.5 \\ \cos \theta_{0} & -0.235 \pm 0.037 & -0.196 \pm 0.021 & -0.163 \pm 0.029 & -0.203 \pm 0.029 & -0.167 \pm 0.026 & -0.174 \pm 0.019 \\ (1- & (1.88 \pm 0.06) & (1.63 \pm 0.04) \times & (1.39 \pm 0.04) \times & (1.34 \pm 0.03) \times & (1.20 \pm 0.02) \times & (1.04 \pm 0.02) \times \\ \left.\cos \theta_{0}\right) / V_{\mathrm{Th}}{ }^{2} & \times 10^{-5} & 10^{-5} & 10^{-5} & 10^{-5} & 10^{-5} & 10^{-5}\end{array}$




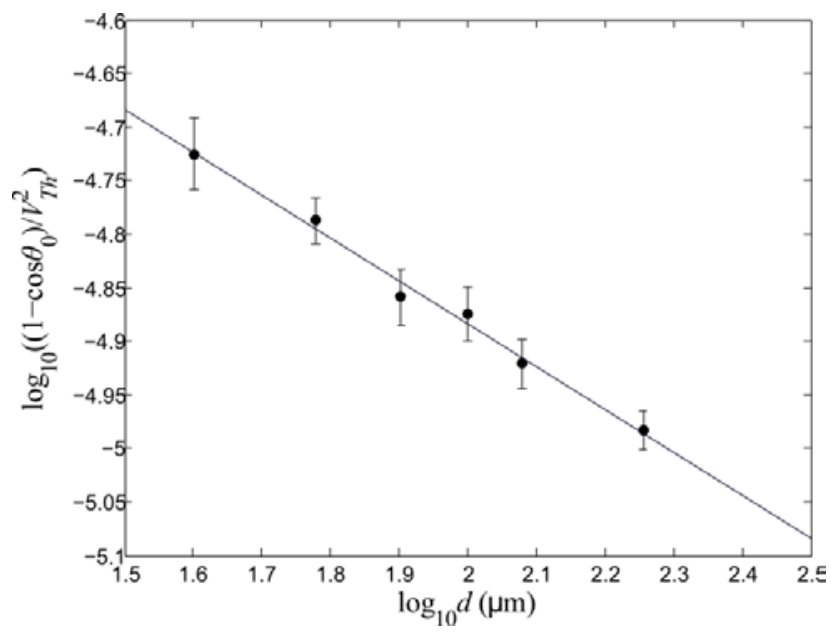

Figure 6. Experimentally determined dependence of $\log _{10}\left(\left(1-\cos \theta_{0}\right) / V_{T h}^{2}\right)$ on $\log _{10} d$.

Figure 6 shows that the dependence (log base 10) of the slopes in the insets in Figure 5, i.e. $\log 10\left(\left(1-\cos \theta_{0}\right) / V_{T h}{ }^{2}\right)$, on the electrode size (log base 10), i.e. $\log _{10} d$, is linear. The best fit equation is $\log _{10}\left(\left(1-\cos \theta_{0}\right) / V_{T h}{ }^{2}\right)=-0.400 \log _{10} d-4.080$; the slope is obtained exactly as (-

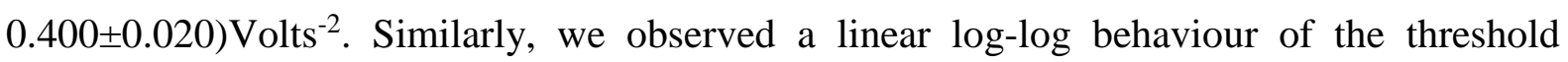
voltage with a best fit relationship, $\log _{10} V_{T h}=0.185 \log _{10} d+2.110$. Thus, all our data, shown in Figure 5, can be scaled using a dependence on $V_{r m s}{ }^{2} / d^{0.400}$ as shown in Figure 7. Plots of contact angle-voltage relationship and $\Delta \cos \theta=\cos \theta\left(V_{r m s}\right)-\cos \theta(0)$ versus the (rms) voltage squared together with the scaled data are provided in Supplementary information (Figure S4a, Figure S4b, respectively).

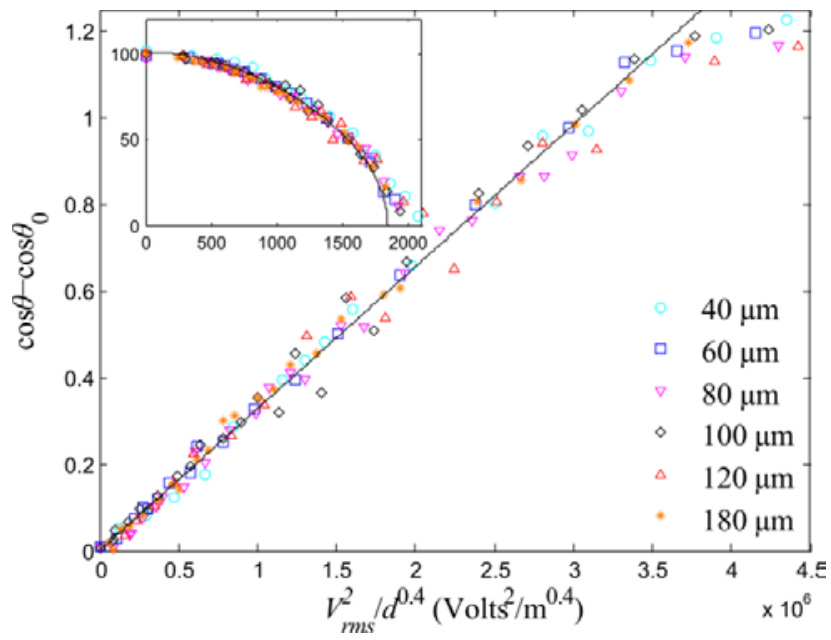


Figure7. Data plotted as $\Delta \cos \theta=\cos \theta\left(V_{r m s}\right)$ - $\cos \theta(0)$ versus (rms) voltage squared, scaled on the master curve. Inset: Scaled contact angle-voltage dependence.

In these experiments, the scaling of the strength of the dielectrowetting with electrode size is $\sim 1 / d^{0.4}$. Thus, the strength of the dielectrowetting effect on droplets using the four-phase activated spiral electrode pattern size differs from the $1 / d$ scaling of stripe droplets previously observed using the straight interdigitated electrodes. ${ }^{30,31}$ In dimensional terms, equations (2) and (3) suggest there must be additional physical dependence which would scale as $1 / d^{0.6}$ influencing the strength of the dielectrowetting. In these spiral electrode pattern designs, the most obvious physical parameter would be related to the length of the spiral contacted by the liquid. This lays the experimental basis for future theoretical calculations to clarify the relationship between electrode pattern design and actuation, and the resulting electric field distribution and its influence on the total energy of a droplet including interfacial energies. We believe that our demonstration of the possibility of removing the stripe limitation in dielectrowetting may inspire other types of electrode designs and alternative approaches to achieve axisymmetric droplet spreading using dielectrowetting to be developed in the future. More generally, it is interesting that dielectrowetting, which manipulates the diploes of a dielectric liquid, can achieve low contact angles and film states, but electrowetting, which manipulates the electrical charges of a conducting liquid, cannot. This may help understand the origin of such saturation effects in electrowetting. ${ }^{11,23}$

\section{CONCLUSION}

Our experiments show that it is possible to spread a droplet of an isotropic dielectric liquid in a near axisymmetric manner by using spiral electrodes and four-phase electric signals with successive $90^{\circ}$ phase shifts. The results also show how liquid dielectrophoresis can be used to reduce the contact angle of a droplet in a voltage-controlled manner without needing to contact the droplet directly. The results also show that it is possible to obtain a sequence of 
partial wetting states concluding with a film state, which it has not been possible to observe in literature studies on electrowetting. The ability to control the contact angle by dielectrowetting has significance for processes such as droplet-based microfluidics, ${ }^{42}$ lab-ona-chip systems, ${ }^{8}$ liquid-based optics, ${ }^{16,17,36}$ coating and other processes where enhanced or controlled spreading are desired. It is also relevant for any process involving control of films, such as in inking and painting where controlled spreading of droplets may be beneficial, or in high speed coating involving forced wetting ${ }^{43}$ where increasing the wetting delays the beginning of bubble entrainment. ${ }^{44,45}$ Finally, it is possible that dielectrowetting could be combined with superoleophobicity to control of non-aqueous droplets in air across the full contact angle range keeping the near axisymmetric circular shape of the droplet when viewed from above that is essential for developing dielectric liquid lenses. ${ }^{26,36}$

\section{AUTHOR INFORMATION}

\section{Corresponding Author}

*E-mail: z.brabcova@northumbria.ac.uk; glen.mchale@northumbria.ac.uk

\section{Notes}

The authors declare no competing financial interest.

\section{ACKNOWLEDGEMENTS}

The work was performed under the umbrella of COST Action MP1106 (Smart and Green Interfaces) and financially supported by EPSRC grants EP/K014803/1 and EP/K015192/1. The authors also acknowledge Dr Michael Cooke, Dr Pietro Maiello, Dr. Edward Bentley, Dr. Ben Xu and Dr. Ariosto Matranga for valuable advice and technical support.

\section{REFERENCES}

(1) Bonn, D.; Eggers, J.; Indekeu, J.; Meunier, J. Wetting and Spreading. Rev. Mod. Phys. 2009, 81 (2), 739-805. 
(2) Myers, D. Surfactant Science and Technology, 3rd Editio.; John Wiley \& Sons, Inc: New Jersey, 2006.

(3) Yuan, Y.; Lee, T. R. Contact Angle and Wetting Properties. In Surface Science Techniques; 2013; Vol. 51, pp 1-34.

(4) Bracco, G.; Holst, B. Surface Science Techniques; Springer, 2013; Vol. 51.

(5) Lee, J.; Moon, H.; Fowler, J.; Schoellhammer, T.; Kim, C. Electrowetting and Electrowetting-on-Dielectric for Microscale Liquid Handling. Sensors Actuators A Phys. 2002, 95, 259-268.

(6) Pollack, M. G.; Fair, R. B.; Shenderov, A. D. Electrowetting-Based Actuation of Liquid Droplets for Microfluidic Applications. Appl. Phys. Lett. 2000, 77 (82), 1725201107.

(7) Paik, P.; Pamula, V. K.; Pollack, M. G.; Fair, R. B. Electrowetting-Based Droplet Mixers for Microfluidic Systems. Lab Chip 2003, 28-33.

(8) Jones, T. B. Liquid Dielectrophoresis on the Microscale. J. Electrostat. 2001, 52, 290 299.

(9) Jones, T. B. On the Relationship of Dielectrophoresis and Electrowetting. Langmuir 2002, 18, 4437-4443.

(10) Berge, B. Electrocapillarity and Wetting of Insulator Films by Water. In Academie des Sciences, Serie II: Mecanique; 1993; Vol. 317, pp 157-163.

(11) Mugele, F.; Baret, J.-C. Electrowetting: From Basics to Applications. J. Phys. Condens. Matter 2005, 17 (28), R705-R774.

(12) Shamai, R.; Andelman, D.; Hayes, R. Water, Electricity, and between ... On Electrowetting and Its Applications. Soft Matter 2008, 4, 38-45.

(13) Xu, M.; Xu, D.; Ren, H.; Yoo, I.-S.; Wang, Q.-H. An Adaptive Liquid Lens with Radial Interdigitated Electrode. J. Opt. 2014, 16, 105601.

(14) Berge, B.; Peseux, J. Variable Focal Lens Controlled by an External Voltage: An Application of Electrowetting. Eur. Phys. J. E 2000, 3, 159-163.

(15) Kuiper, S.; Hendriks, B. H. W. Variable-Focus Liquid Lens for Miniature Cameras. Appl. Phys. Lett. 2004, 85 (7), 1128-1130.

(16) Russell, A.; Kreit, E.; Heikenfeld, J. Scaling Dielectrowetting Optical Shutters to Higher Resolution: Microfluidic and Optical Implications. Langmuir 2014, 30, 53575362.

(17) Zhao, R.; Cumby, B.; Russell, A.; Heikenfeld, J. Large Area and Low Power Dielectrowetting Optical Shutter with Local Deterministic Fluid Film Breakup. Appl. Phys. Lett. 2013, 103, 1-5.

(18) Darhuber, A. A.; Valentino, J. P.; Davis, J. M.; Troian, S. M.; Wagner, S. Microfluidic Actuation by Modulation of Surface Stresses. Appl. Phys. Lett. 2003, 82 (77).

(19) Yi, U.-C.; Kim, C.-J. Characterization of Electrowetting Actuation on Addressable Single-Side Coplanar Electrodes. J. Micromechanics Microengineering 2006, 16 (10), 2053-2059.

(20) Banpurkar, A. G.; Nichols, K. P.; Mugele, F. Electrowetting-Based Microdrop Tensiometer. Langmuir 2008, 24 (19), 10549-10551. 
(21) Heikenfeld, J.; Dhindsa, M. Electrowetting on Superhydrophobic Surfaces: Present Status and Prospects. J. Adhes. Sci. Technol. 2008, 22, 319-334.

(22) Quinn, A.; Sedev, R.; Ralston, J. Contact Angle Saturation in Electrowetting. J. Phys. Chem. B 2005, 109, 6268-6275.

(23) Chevalliot, S.; Kuiper, S.; Heikenfeld, J. Experimental Validation of the Invariance of Electrowetting Contact Angle Saturation. J. Adhes. Sci. Technol. 2012, 26, 12-17.

(24) Mugele, F. Fundamental Challenges in Electrowetting: From Equilibrium Shapes to Contact Angle Saturation and Drop Dynamics. Soft Matter 2009, 5, 3377-3384.

(25) Lewpiriyawong, N.; Yang, C.; Lam, Y. C. Dielectrophoretic Manipulation of Particles in a Modified Microfluidic H Filter with Multi-Insulating Blocks. Biomicrofluidics 2008, 2 (3).

(26) Brown, C. V; Wells, G. G.; Newton, M. I.; Mchale, G. Voltage-Programmable Liquid Optical Interface. Nat. Photonics 2009, 3 (420), 8-10.

(27) Pellat, H. Mesure de La Force Agissant Sur Les Dielectriques Liquides Non Electrises Places Dans Un Champ Electrique. C. R. Acad. Sci. Paris 1895, 119, 691-694.

(28) Pohl, H. A. Dielectrophoresis: The Behaviour of Neutral Matter in Non-Uniform Electric Fields. In Cambridge Monographs on Physics; Cambridge Univ. Press, 1978.

(29) Jones, T. B.; Gunji, M.; Washizu, M.; Feldman, M. J. Dielectrophoretic Liquid Actuation and Nanodroplet Formation. J. Appl. Phys. 2001, 89 (77), 1441-1725.

(30) McHale, G.; Brown, C. V; Newton, M. I.; Wells, G. G.; Sampara, N. Dielectrowetting Driven Spreading of Droplets. Phys. Rev. Lett. 2011, 107 (186101), 1-4.

(31) McHale, G.; Brown, C. V; Sampara, N. Voltage Induced Spreading and Superspreading of Liquids. Nat. Commun. 2013, 4 (1605), 1-19.

(32) Brown, C. V; McHale, G.; Trabi, C. L. Dielectrophoresis-Driven Spreading of Immersed Liquid Droplets. Langmuir 2015, 31, 1011-1016.

(33) Shirtcliffe, N. J.; Mchale, G.; Newton, M. I. The Superhydrophobicity of Polymer Surfaces : Recent Developments. J. Polym. Sci. Part B Polym. Phys. 2011, 49 (17), 1203-1217.

(34) Edwards, A. M. J.; Ledesma-Aguilar, R.; Newton, M. I.; Brown, C. V.; Mchale, G. Not Spreading in Reverse: The Dewetting of a Liquid Film into a Single Droplet. Submitt. to Sci. Adv. 2016.

(35) Morgan, H.; Garc, A.; Bakewell, D. The Dielectrophoretic and Travelling Wave Forces Generated by Interdigitated Electrode Arrays : Analytical Solution Using Fourier Series. J. Phys. D. Appl. Phys. 2001, 34, 1553-1561.

(36) McHale, G.; Brown, C. V; Newton, M. I.; Wells, G. G.; Sampara, N. Developing Interface Localized Liquid Dielectrophoresis for Optical Applications. Opt. Des. Test. V 2012, 8557, 1-8.

(37) Cheng, C.; Chang, C. A.; Yeh, J. A. Variable Focus Dielectric Liquid Droplet Lens. Opt. Express 2006, 14 (9), 4101-4106.

(38) Sampara, N. Voltage Induced Spreading and Liquid Optical Devices, Nottingham Trent University, 2013.

(39) Dalton, C.; Goater, A. D.; Burt, J. P. H.; Smith, H. V. Analysis of Parasites by 
Electrorotation. J. Appl. Microbiol. 2004, 96 (1), 24-32.

(40) Goater, A. D.; Burt, J. P. H.; Pethig, R. A Combined Travelling Wave Dielectrophoresis and Electrorotation Device : Applied to the Concentration and Viability Determination of Cryptosporidium. J. Phys. D. Appl. Phys. 1997, 30, 65-69.

(41) Kajiya, T.; Schellenberger, F.; Papadopoulos, P.; Vollmer, D.; Butt, H.-J. 3D Imaging of Water-Drop Condensation on Hydrophobic and Hydrophilic Lubricant-Impregnated Surfaces. Sci. Rep. 2016, 6, 1-10.

(42) Pollack, M. G.; Fair, R. B.; Carolina, N. Electrowetting-Based Actuation of Liquid Droplets for Microfluidic Applications. 2000, 77 (11), 1725-1726.

(43) Liu, F.; Shen, W. Forced Wetting and Dewetting of Liquids on Solid Surfaces and Their Roles in Offset Printing. Colloids Surfaces A Physicochem. Eng. Asp. 2008, 316, 62-69.

(44) Blake, T. D. Forced Wetting of a Reactive Surface. Adv. Colloid Interface Sci. 2012, 179-182, 22-28.

(45) Blake, T. D. The Physics of Moving Wetting Lines. J. Colloid Interface Sci. 2006, 299 (1), 1-13. 